\title{
Ectopic fat accumulation in patients with COPD: an ECLIPSE substudy
}

\author{
This article was published in the following Dove Press journal: \\ International Journal of COPD \\ 31 January 2017 \\ Number of times this article has been viewed
}

\section{Mickaël Martin,' Natalie \\ Almeras,' Jean-Pierre \\ Després,' Harvey $O$ \\ Coxson, ${ }^{2}$ George R \\ Washko, ${ }^{3}$ Isabelle \\ Vivodtzev, ${ }^{4}$ Emiel FM \\ Wouters, ${ }^{5}$ Erica Rutten, ${ }^{6}$ \\ Michelle CWilliams, ${ }^{7}$ John \\ T Murchison, ${ }^{8}$ William \\ MacNee, ${ }^{7}$ Don D Sin, ${ }^{2}$ \\ François Maltais' \\ On behalf of the Evaluation \\ of COPD Longitudinally \\ to Identify Predictive \\ Surrogate Endpoints \\ (ECLIPSE) Study Group}

'Research Centre, Institut universitaire de cardiologie et de pneumologie de Québec, Université Laval, Québec, QC, ${ }^{2}$ Department of Radiology, University of British Columbia, Vancouver, BC, Canada; ${ }^{3}$ Department of Medicine, Brigham and Women's Hospital, Harvard Medical School, Boston, MA, USA; ${ }^{4}$ Hypoxia Pathophysiology Laboratory, Grenoble University Hospital, Grenoble, France; ${ }^{5}$ Department of Respiratory Medicine, Maastricht University Medical Center, Maastricht, ${ }^{6}$ Research and Development, CIRO, Horn, the Netherlands; ${ }^{7}$ Department of Respiratory Medicine, University of Edinburgh, ${ }^{8}$ Department of Radiology, Royal Infirmary of Edinburgh, Edinburgh, UK

Correspondence: François Maltais Centre de Pneumologie, Institut universitaire de cardiologie et de pneumologie de Québec, 2725

Chemin Sainte-Foy, Québec, QC, Canada GIV 4G5

Fax +I 4186564762

Email francois.maltais@fmed.ulaval.ca
Background: Obesity is increasingly associated with COPD, but little is known about the prevalence of ectopic fat accumulation in COPD and whether this can possibly be associated with poor clinical outcomes and comorbidities. The Evaluation of COPD Longitudinally to Identify Predictive Surrogate Endpoints (ECLIPSE) substudy tested the hypothesis that COPD is associated with increased ectopic fat accumulation and that this would be associated with COPD-related outcomes and comorbidities.

Methods: Computed tomography (CT) images of the thorax obtained in ECLIPSE were used to quantify ectopic fat accumulation at L2-L3 (eg, cross-sectional area [CSA] of visceral adipose tissue [VAT] and muscle tissue [MT] attenuation, a reflection of muscle fat infiltration) and CSA of MT. A dose-response relationship between CSA of VAT, MT attenuation and CSA of MT and COPD-related outcomes (6-minute walking distance [6MWD], exacerbation rate, quality of life, and forced expiratory volume in 1 second $\left[\mathrm{FEV}_{1}\right]$ decline) was addressed with the Cochran-Armitage trend test. Regression models were used to investigate possible relationships between CT body composition indices and comorbidities.

Results: From the entire ECLIPSE cohort, we identified 585 subjects with valid CT images at L2-L3 to assess body composition. CSA of VAT was increased $(P<0.0001)$ and MT attenuation was reduced (indicating more muscle fat accumulation) in patients with $\operatorname{COPD}(P<0.002)$. Progressively increasing CSA of VAT was not associated with adverse clinical outcomes. The probability of exhibiting low 6MWD and accelerated $\mathrm{FEV}_{1}$ decline increased with progressively decreasing MT attenuation and CSA of MT. In COPD, the probability of having diabetes $(P=0.024)$ and gastroesophageal reflux $(P=0.0048)$ at baseline increased in parallel with VAT accumulation, while the predicted MT attenuation increased the probability of cardiovascular comorbidities $(P=0.042)$. Body composition parameters did not correlate with coronary artery scores or with survival.

Conclusion: Ectopic fat accumulation is increased in COPD, and this was associated with relevant clinical outcomes and comorbidities.

Keywords: metabolic syndrome, obesity, abdominal adiposity, muscle

\section{Introduction}

Obesity is becoming a major health issue in patients with COPD, as it is in the general population. Several studies have reported an increased proportion of patients with COPD who are either overweight or obese. ${ }^{1-4}$ Considering the well-established link between obesity and hypertension, type 2 diabetes, cancer and cardiovascular diseases (CVDs) ${ }^{5}$ and the fact that COPD is an independent risk factor for CVDs, ${ }^{6}$ this increased prevalence of obesity in COPD is a source of concern. Consistent with these observations, all-cause mortality and cardiovascular mortality are also increased in patients with COPD. ${ }^{7}$ Depending on COPD severity, CVDs are the primary cause of mortality in $20 \%-27 \%$ of patients with COPD. ${ }^{8,9}$ 
One important issue to consider is that body mass index (BMI) is only a crude index of body composition while not allowing the quantification of ectopic fat accumulation. Rather than body size, body shape may be important to consider in the interpretation of the relationship between obesity and COPD. For instance, it is very well documented that the adverse metabolic and cardiovascular consequences of obesity are largely explained by the presence of unwanted adipose tissue (AT) accumulation in the abdomen and in normally lean tissues such as the liver, the heart and the skeletal muscles (collectively called ectopic fat tissue accumulation). ${ }^{10}$ Individuals with a selective accumulation of ectopic fat are at increased risk of developing features of the metabolic syndrome, diabetes, an atherogenic dyslipidemia, or CVDs compared to those who preferentially accumulate lipids in subcutaneous "healthy" AT. ${ }^{10}$

We took advantage of the Evaluation of COPD Longitudinally to Identify Predictive Surrogate Endpoints (ECLIPSE) cohort with a 3-year follow-up to address the following questions: 1) Is COPD associated with increased ectopic fat accumulation within the abdomen and in the muscles? 2) Does ectopic fat accumulation modify the expression of COPD in being associated with reduced functional and health status, as well as with forced expiratory volume in 1 second $\left(\mathrm{FEV}_{1}\right)$ rate of decline, exacerbations, mortality and cardiovascular comorbidities? Our overarching hypothesis was that COPD would be associated with increased ectopic fat accumulation compared to controls with normal lung function and that this would be associated with adverse clinical outcomes.

\section{Methods}

\section{Study design and patients}

ECLIPSE (ClinicalTrials.gov, NCT00292552) was a multicenter, observational and longitudinal study to identify surrogate markers of COPD progression. ${ }^{11}$ It enrolled 2,164 current or ex-smokers ( $\geq 10$ pack-years) with Global Initiative for Chronic Obstructive Lung Disease (GOLD) stages 2-4, 337 smokers with normal lung function and 245 neversmokers. ${ }^{12}$ All ECLIPSE participants underwent a low-dose volumetric computed tomography (CT) scan of the chest at baseline. In a subset of them that constitutes the population for the current report, a valid CT scan slice at L2-L3 could be identified, allowing to assess body composition from which fat and muscle densities, a reflection of tissue fatty infiltration, could be accurately quantified. ${ }^{13}$

Patients' characteristics (age, race, sex, smoking history), chest CT scan, pulmonary function tests, St Georges' Respiratory Questionnaire (SGRQ) score and 6-minute walking distance (6MWD) were obtained at baseline and yearly for the 3 -year study duration as previously reported. ${ }^{11,14,15}$ Standardized questionnaires were used to record comorbidity at baseline. COPD exacerbations and mortality were also recorded. All patients provided written informed consent, and the study was approved by the ethics committees of the participating centers. The ECLIPSE steering and scientific committees approved the protocol for this substudy (Table S1).

\section{Body composition and coronary artery calcification assessment}

All ECLIPSE participants underwent a low-dose volumetric CT scan of the chest at baseline with the following protocol: $120-\mathrm{kV}$ peak, $40 \mathrm{~mA}$, and 1.00- or 1.25-mm slice thickness, at full inspiration. For the purpose of this ECLIPSE substudy, we analyzed all subjects with a thoracic CT scan that contained images at L2-L3 intervertebral space. Ectopic fat accumulation was quantified by measuring cross-sectional area (CSA) of visceral adipose tissue (VAT) and muscle tissue (MT) attenuation, a reflection of muscle fat infiltration. Subcutaneous adipose tissue (SCAT) and CSAs of MT were also assessed. Measures of fat and MT (psoas, paraspinal and abdominal wall muscles) CSA and attenuation were performed by the same investigator (MM) using the body composition analysis platform of the Institut universitaire de cardiologie et de pneumologie de Québec and a specialized image analysis software (sliceOmatic; TomoVision, Montreal, QC, Canada) according to standardized techniques (Figure 1). ${ }^{13}$ CSAs of AT and MT were measured using an attenuation range of -190 to -30 Hounsfield units (HUs) and -29 to $130 \mathrm{HUs}$, respectively. The mean attenuation value in HU of each structure was generated. To avoid subjectivity in the separation between SCAT and VAT, these two fat tissues were separated from each other midway in the thickness of abdominal wall muscles. Subjects for whom a tissue surface area $>1 \mathrm{~cm}^{2}$ fell outside the field of view of the scope were excluded from further analysis. A random selection of $10 \%$ of all segmented images was redone by the same evaluator to assess for intraobserver variation. Interobserver variation was evaluated on a random sample of $10 \%$ of the segmented images that were also analyzed by a second imaging expert. We also took advantage of the thoracic CT scan to quantify coronary artery calcification, a surrogate of coronary artery disease, using standardized techniques. ${ }^{16}$

\section{Statistical analyses}

Characteristics were compared between patients with COPD and controls (smokers with normal lung function and never-smokers), using Student's $t$-test statistics for 


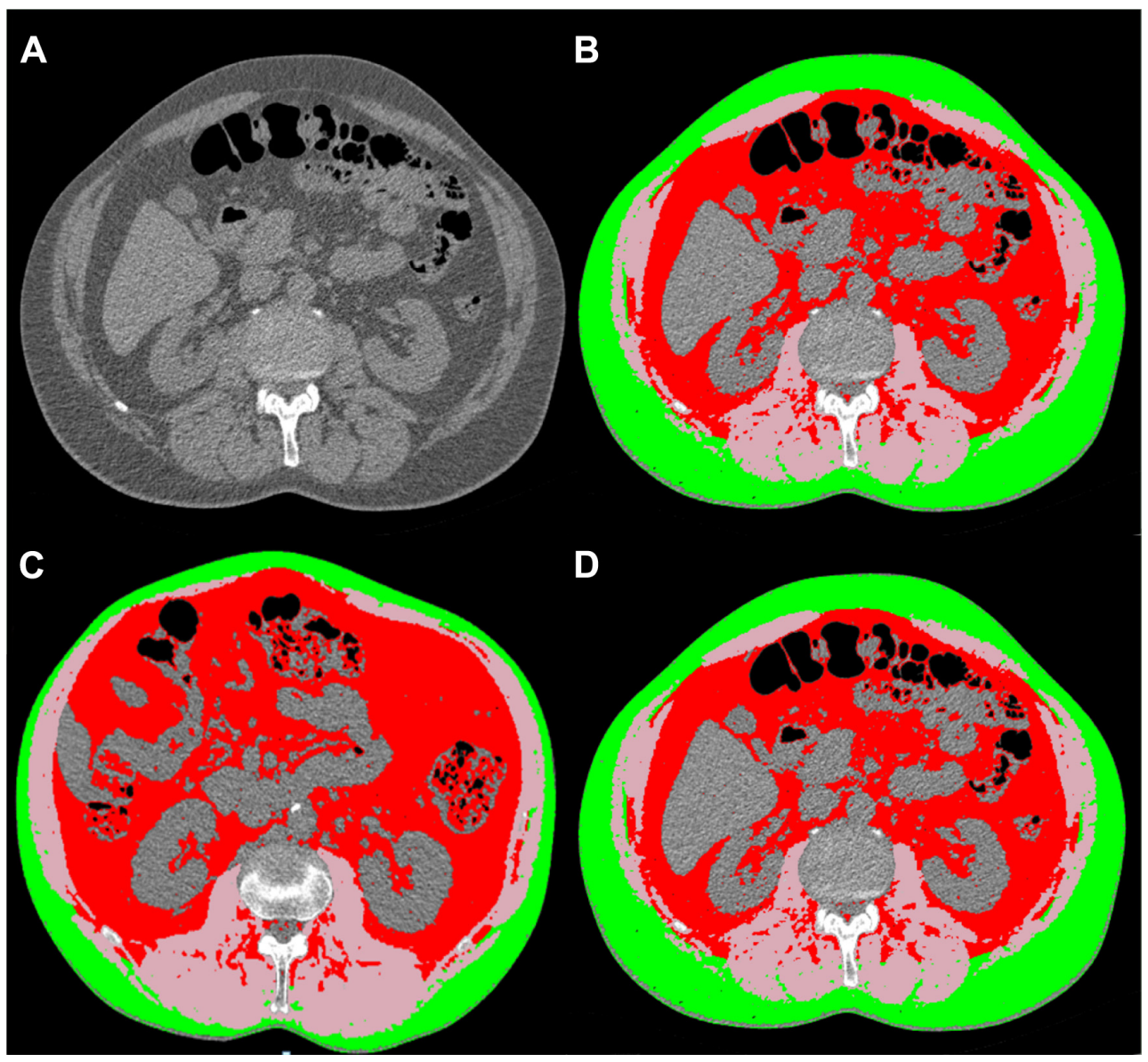

Figure I Body composition analysis and differences in fat distribution.

Notes: Examples of a CT slice at L2-L3 before (A) and after segmentation (B). An example is also provided for two patients with COPD with same BMI and similar waist circumference but marked difference in body composition, with one patient (C) having more VAT (shown in red) than the other patient (D). After segmentation, SCAT was identified in green, VAT in red and MT in pink. CSAs of tissues $\left(\mathrm{cm}^{2}\right)$ were computed on the segmented structures using an attenuation range of -190 to -30 HUs for AT and -29 to $130 \mathrm{HUs}$ for MT. The mean attenuation value in $\mathrm{HU}$ of each structure was also quantified.

Abbreviations: CT, computed tomography; BMI, body mass index; SCAT, subcutaneous adipose tissue; VAT, visceral adipose tissue; MT, muscle tissue; CSA, crosssectional area; HU, Hounsfield unit; AT, adipose tissue.

continuous variables and chi-square statistics for discrete variables. Coronary artery calcification scores were analyzed on a $\log _{10}$ scale because of their lognormal distribution. Patients with COPD were further classified according to the GOLD spirometric classification system. ${ }^{17}$ The overall test-retest reproducibility of each body composition parameter was assessed by calculating a Pearson's correlation coefficient between evaluation 1 and evaluation 2 for each CT body composition parameter. Test-retest reliability was evaluated using intraclass correlation coefficient (ICC) as it reflects both systematic and random differences in test measures. ${ }^{18}$ An ICC $>0.75$ was judged to be excellent. ${ }^{19}$ CT body composition variables were compared between patients with COPD and controls, and also across GOLD spirometric grades, using a multivariate analysis of covariance (MANCOVA) model, with age, sex, BMI and smoking status included as covariates. When a significant effect of groups or subgroups were identified, univariate analysis of covariance (ANCOVA) models were adjusted to investigate which body composition variables were different, and least significant difference-protected multiple comparisons were performed to differentiate the subgroups.

The association between body composition parameters with clinical outcomes of interest (6MWD, exacerbation rate, quality of life, $\mathrm{FEV}_{1}$ decline) was addressed using the Cochran-Armitage trend test to determine a dose-response relationship between CSA of VAT, mean MT attenuation, CSA of MT and each of the clinical outcomes. For this analysis, body composition parameters (explanatory variables) were expressed in four equal groups determined by their quartiles. In this analysis, quartile 1 was considered as the reference value, while quartiles 2, 3 and 4 indicated progressive increase in ectopic fat accumulation or decrease in CSA of MT. Each patient-reported outcome was expressed as dichotomous variables using specific cut-points: $350 \mathrm{~m}$ for the $6 \mathrm{MWD},{ }^{20}$ more than or equal to 
two exacerbations per year, ${ }^{17}$ SGRQ score $>25^{17}$ and $\mathrm{FEV}_{1}$ decline $>40 \mathrm{~mL} /$ year. ${ }^{21}$ The impact of body composition on comorbidities was assessed by logistic regression models, while its relationship with survival was studied using a Cox proportional hazards regression model. All analyses were controlled for age, sex, BMI, smoking status and $\mathrm{FEV}_{1}$. $P$-values $<0.05$ were considered statistically significant. Data analyses were performed using SAS version 9.4 (SAS Institute Inc., Cary, NC, USA).

\section{Results}

We identified 585 patients (511 patients with COPD and 74 controls) for whom a CT slice at L2-L3 could be analyzed for body composition assessment. Patients' characteristics are outlined in Table 1. Patients with COPD and controls had similar BMI but important differences were otherwise noted: patients with COPD were older, had a higher proportion of men and were current/former smokers compared to controls. They also exhibited higher SGRQ scores (worse healthrelated quality of life), greater prevalence of cardiovascular comorbidities (angina, heart attack, myocardial infarction, stroke and heart failure), hypertension and greater coronary artery calcification score than controls. During the 3-year follow-up, 50 deaths were recorded (49 patients with COPD and one control), resulting in lower mean survival days in patients with COPD. A total of 1,661 moderate-to-severe exacerbations were reported in patients with COPD, with a mean exacerbation rate of $1.28 \pm 0.06 /$ year/patient (range: 0-9/year/patient).

The Pearson's coefficient and ICC for each body composition parameter are reported in Table 2 and show an excellent intra- and inter-observer reproducibility of our body composition measurements.

CT body composition data adjusted for age, sex, smoker status and BMI are presented in Figure 2. For a similar BMI (Figure 2A), patients with COPD exhibited higher CSA of VAT (Figure 2B), lower MT attenuation (Figure 2C) and a tendency to have lower CSA of MT (Figure 2D) than controls. CSA of SCAT could be quantified in 384 subjects (327 COPD subjects and 57 controls) in whom the abdomen external wall

Table I Baseline characteristics by group

\begin{tabular}{|c|c|c|c|}
\hline Variables & $\operatorname{COPD}(n=5 \mid I)$ & Controls $(n=74)$ & $P$-value \\
\hline Age (years) & $63.8 \pm 6.9$ & $56.8 \pm 10.0$ & $<0.000$ I \\
\hline BMI $\left(\mathrm{kg} / \mathrm{m}^{2}\right)$ & $24.4 \pm 5.0$ & $25.3 \pm 4.3$ & 0.1761 \\
\hline \multicolumn{4}{|l|}{ Sex } \\
\hline Male, n (\%) & $314(6 I)$ & $30(4 I)$ & \multirow[t]{2}{*}{0.0007} \\
\hline Female, n (\%) & $197(39)$ & $44(59)$ & \\
\hline \multicolumn{4}{|l|}{ Smoking status } \\
\hline Current smokers, n (\%) & $201(39)$ & $20(27)$ & \multirow[t]{3}{*}{$<0.000$ I } \\
\hline Former smokers, n (\%) & $310(6 I)$ & $17(23)$ & \\
\hline Never-smokers, n (\%) & $0(0)$ & $37(50)$ & \\
\hline Pack/year & $48.7 \pm I . I$ & $14.9 \pm 4.4$ & $<0.000$ I \\
\hline \multicolumn{4}{|l|}{ Pulmonary function } \\
\hline $\mathrm{FEV}_{1}$ (\% predicted value) & $40.7 \pm 15.3$ & $108.3 \pm 13.2$ & $<0.000$ I \\
\hline $\mathrm{FVC}(\mathrm{L})$ & $2.7 I \pm 0.9$ & $4.07 \pm 0.9$ & $<0.000$ I \\
\hline $\mathrm{FEV}_{1} / \mathrm{FVC}$ & $0.4 I \pm 0.0 I$ & $0.79 \pm 0.01$ & $<0.000$ I \\
\hline FEV , decline (L/year) & $0.052 \pm 0.023$ & NA & NA \\
\hline \multicolumn{4}{|l|}{ Clinical variables } \\
\hline SGRQ total score & $48.4 \pm 17.2$ & $9.5 \pm 9.7$ & $<0.000$ I \\
\hline 6MWD $(\mathrm{m})$ & $366.3 \pm 5.7$ & NA & NA \\
\hline Exacerbation number (number/years) & $1.3 \pm 0.1$ & $0(0)$ & $<0.000$ I \\
\hline Mortality, n (\%) & $49(9.5)$ & I (I.3) & 0.02 \\
\hline Mean survival (days) & $1,016 \pm 6$ & $1,060 \pm 5$ & $<0.01$ \\
\hline \multicolumn{4}{|l|}{ Comorbidities } \\
\hline Cardiovascular ${ }^{\dagger}, \mathrm{n}(\%)$ & $255(50)$ & $\mathrm{I}(\mathrm{I})$ & 0.0078 \\
\hline Hypertension, n (\%) & $175(34)$ & $9(12)$ & 0.0001 \\
\hline CAC score, Agatston units & $540 \pm 45$ & $251 \pm 120$ & 0.0005 \\
\hline Diabetes, $\mathrm{n}(\%)$ & $31(6)$ & $\mathrm{I}(\mathrm{I})$ & 0.1068 \\
\hline Reflux/heartburn, n (\%) & $110(22)$ & $15(20)$ & 0.8802 \\
\hline
\end{tabular}

Notes: Data shown as mean \pm SE or $\mathrm{n}(\%)$. Includes angina, heart attack, myocardial infarction, stroke and heart failure.

Abbreviations: BMI, body mass index; FEV , forced expiratory volume in I second; FVC, forced vital capacity; NA, not applicable; SGRQ, St George's Respiratory Questionnaire; 6MWD, 6-minute walking distance; CAC, coronary artery calcification; SE, standard error. 
Table 2 Internal validity and external validity of the CT image analysis

\begin{tabular}{|c|c|c|c|c|}
\hline \multirow[t]{2}{*}{ Variables } & \multicolumn{2}{|c|}{ Internal validity } & \multicolumn{2}{|c|}{ External validity } \\
\hline & \multicolumn{2}{|c|}{ Pearson's $r$ Intraclass $r$} & \multicolumn{2}{|c|}{ Pearson's $r$ Intraclass $r$} \\
\hline VAT surface & 1.00 & 1.00 & 1.00 & 1.00 \\
\hline MT surface & 0.99 & 0.99 & 0.99 & 0.99 \\
\hline MT attenuation & 1.00 & 1.00 & 0.95 & 0.95 \\
\hline SCAT & 1.00 & 1.00 & 0.99 & 0.99 \\
\hline
\end{tabular}

Abbreviations: CT, computed tomography; VAT, visceral adipose tissue; MT, muscle tissue; SCAT, subcutaneous adipose tissue.

fell inside the field of view. SCAT was greater in patients with COPD than in controls (Figure 2E). The same results are also presented according to the severity of airflow obstruction from GOLD stages 2 to 4 . Patients with GOLD 3 and 4 COPDs had lower BMI than GOLD 2 patients, while GOLD 4 patients were characterized by larger CSAs of VAT and SCAT and less MT attenuation than the remaining groups.

The associations between body composition parameters and COPD-associated clinical outcomes are shown in Figure 3. Progressively increasing CSA of VAT was not associated with worsening in functional status (6MWD), exacerbation rate and $\mathrm{FEV}_{1}$ decline but it was associated with a reduced odds ratio of having SGRQ scores $>25$ (indicating worse health status). In contrast, progressively decreasing MT attenuation and CSA of MT were associated with increased probability of exhibiting low 6MWD and accelerated $\mathrm{FEV}_{1}$ decline. In COPD, the probability of having diabetes $(P=0.024)$ and gastroesophageal reflux $(P=0.0048)$ at baseline increased in parallel with VAT accumulation, while MT attenuation predicted increased probability of cardiovascular comorbidities ( $P=0.042$; Figure 4$)$. Body composition parameters did not correlate with coronary artery scores or with survival.

\section{Discussion}

This ECLIPSE substudy reports increased ectopic AT accumulation in a large group of patients with COPD compared to controls with normal lung function. Patients with COPD were characterized by increased CSA of VAT and evidence of muscle fat infiltration as assessed by CT as well as by an increased prevalence of cardiovascular comorbidities. Our study is concordant and extends existing knowledge that patients with COPD are associated with increased VAT accumulation compared to controls $\mathrm{s}^{7,22}$ by reporting, for the first time, decreased MT attenuation (consistent with increased fat infiltration) in COPD and investigating the relationship between ectopic fat accumulation and COPDrelated outcomes and comorbidities. Alterations in CT body composition parameters were particularly notable in patients with GOLD 4 COPD. Interestingly, progressively decreasing MT attenuation and CSA of MT were associated with increased probability of experiencing worse functional status and $\mathrm{FEV}_{1}$ decline and prevalence of CVDs, while VAT accumulation was associated with the prevalence of diabetes and gastroesophageal reflux at study entry. Importantly, these associations remained after adjustment for age, sex, BMI, smoking status and $\mathrm{FEV}_{1}$.

We are not aware of previously published study on intra-abdominal muscle attenuation and CSA data in COPD. Similar to our findings involving the psoas, paraspinal and abdominal wall muscles, evidence of fat infiltration and atrophy of the quadriceps has been reported in COPD. ${ }^{23,24}$ The same authors also reported inverse relationships between indices of quadriceps' fat infiltration and walking capacity in COPD. In this context, it is interesting that lower intraabdominal MT density, a reflection of increased fatty content, and reduced muscle intra-abdominal CSAs were associated with increased probability of having a 6MWD $<350 \mathrm{~m}$. It could be argued that the performance of the psoas and paraspinal muscles that are involved in walking was altered by a combination of fat infiltration and reduced area, together leading to impaired muscle contractility. ${ }^{24,25}$ Alternatively, intra-abdominal muscle fat infiltration and atrophy may simply be a reflection of a generalized process involving several muscle groups, including those of the lower limbs.

The mechanisms for ectopic fat accumulation in COPD were not investigated. These phenomena have been associated with insulin resistance, ${ }^{26}$ resulting from a combination of factors, including age, genetics, poor diet, smoking and lack of vigorous physical activity, to only name a few. ${ }^{10}$ Another potential contributor to ectopic fat accumulation in COPD that was not addressed in the present study included repeated use of systemic corticosteroid to treat COPD exacerbations. ${ }^{27}$ We also did not have direct measurement of physical activity and dietary habits. ${ }^{7}$

Ectopic fat is metabolically active as evidenced by its production of a host of proinflammatory mediators, including TNF- $\alpha$, IL-6, leptin and adiponectin, whose effects may be seen not only locally within the AT but also at the systemic level. Considering systemic inflammation as a risk factor for CVDs and possibly for COPD progression, ${ }^{28,29}$ we speculated that ectopic fat accumulation would be associated with adverse clinical outcomes such as poor functional status and quality of life, increased exacerbation risk and faster $\mathrm{FEV}_{1}$ decline as well as with cardiovascular comorbidities. Although this interpretation is partly supported by 

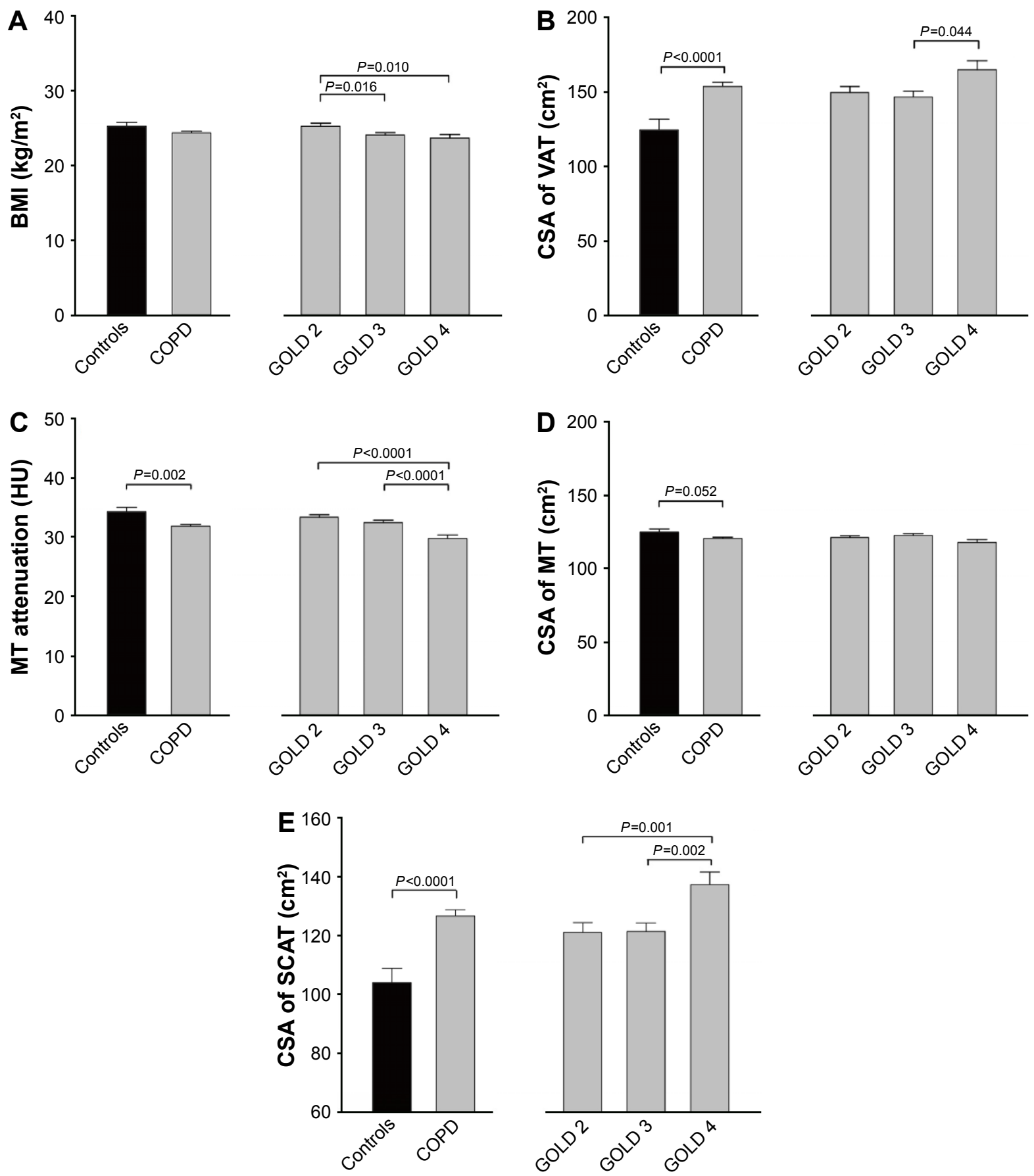

Figure 2 Group mean values \pm SD for BMI (A), CSA of VAT (B), MT attenuation (C), CSA of MT (D) and CSA of SCAT (E) in patients with COPD (gray bars) and in controls (black bars).

Note: Patients with COPD were further classified according to the GOLD spirometric classification system, with a greater score indicating worse airflow obstruction. Abbreviations: BMI, body mass index; CSA, cross-sectional area; VAT, visceral adipose tissue; MT, muscle tissue; SCAT, subcutaneous adipose tissue; GOLD, Global Initiative for Chronic Obstructive Lung Disease; HU, Hounsfield unit; SD, standard deviation.

our data, the reported association between ectopic fat accumulation, clinical outcomes and comorbidities should not be viewed as implying causal relationships. Nevertheless, these associations point to the fact that ectopic fat accumulation could be detrimental to cardiovascular and metabolic health in COPD.
Investigators from the COPDGene study reported a significant association between the amount of VAT and the risk of having a history of a previous myocardial infarction in patients with COPD. ${ }^{30}$ In the present investigation, we found that CSA of VAT and muscle attenuation were, respectively, associated with increased prevalence of diabetes and cardiovascular 


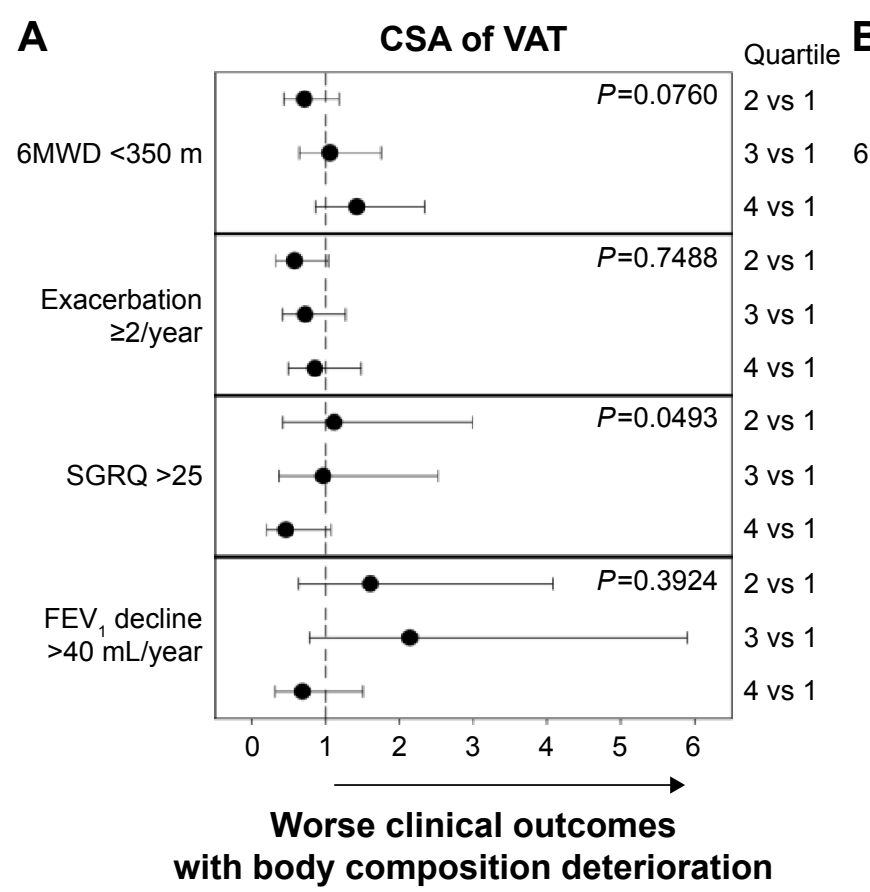

B

$6 \mathrm{MWD}<350 \mathrm{~m}$

Fxacescos

Exacerbation $\geq 2 /$ year

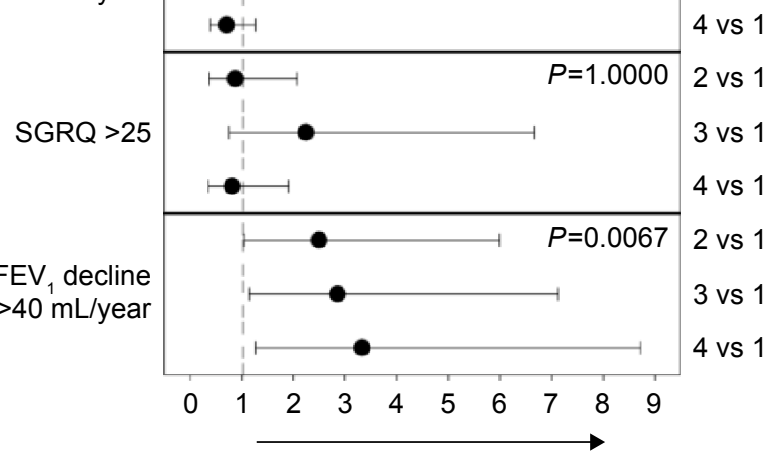

Worse clinical outcomes with body composition deterioration

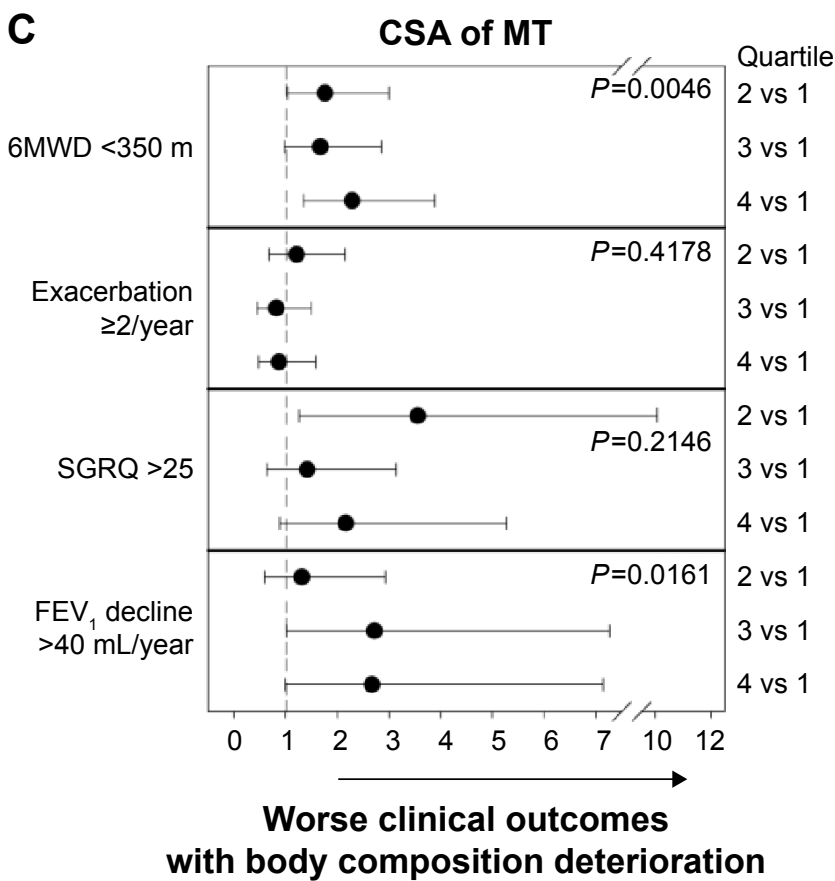

Figure 3 Trend analysis between CT body composition parameters and clinical outcomes in COPD.

Notes: Trend analysis between CSA of VAT (A), MT attenuation (B), and CSA of MT (C), and the 6MWD, exacerbation rate, SGRQ and FEV, decline. For each body composition parameter, the panels report the odds ratio (mean and $95 \% \mathrm{Cl}$ ) of having a worse clinical outcome with the deterioration of the body composition parameter from quartile I (reference) to quartiles 2, 3 and 4. The $P$-values indicate the statistical significance for the trend of the association.

Abbreviations: CT, computed tomography; CSA, cross-sectional area; VAT, visceral adipose tissue; MT, muscle tissue; 6MWD, 6-minute walking distance; SGRQ, St George's Respiratory Questionnaire; FEV , forced expiratory volume in I second; Cl, confidence interval.

comorbidities at study entry. These results are consistent with the bulk of knowledge recognizing ectopic fat accumulation as a risk factor for cardiovascular and metabolic diseases..$^{10} \mathrm{We}$ did not find, however, an association between the extent of coronary artery calcification and VAT in this cohort. This may be an indication that the mechanisms by which VAT contributes to CVD risk do not mainly involve a calcification process.

Our study has several strengths. It involves an appreciable number of patients with longitudinal follow-up, offering the possibility of studying the clinical consequences of ectopic 

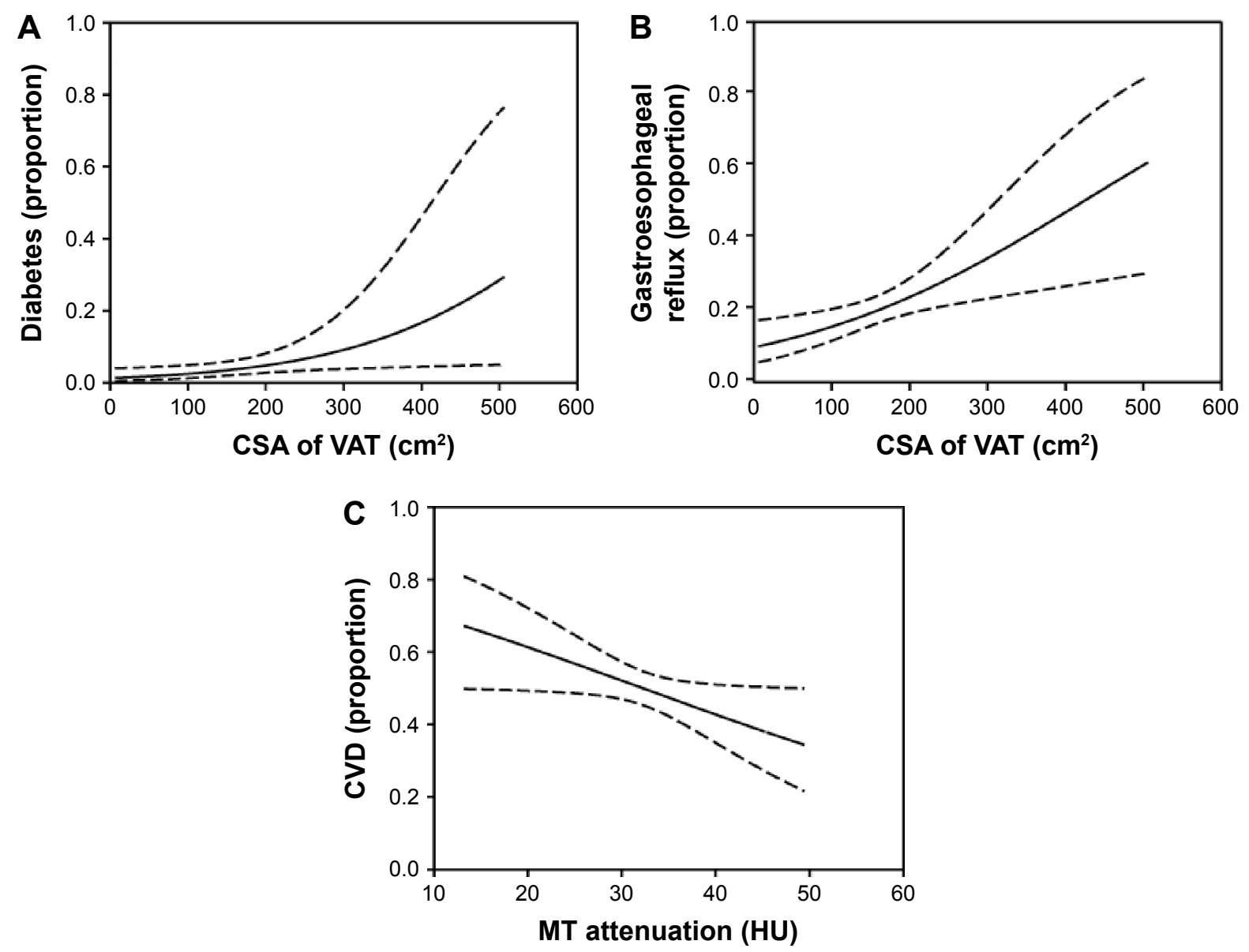

Figure 4 Logistic regression models indicating significant correlations between CT body composition parameters and the proportion of comorbidities for diabetes (A), gastroesophageal reflux (B), and CVD (C) at baseline.

Note: Continuous lines were drawn from the mean regression coefficients with the dashed lines representing the $95 \% \mathrm{Cl}$ of the relationships.

Abbreviations: CT, computed tomography; Cl, confidence interval; VAT, visceral adipose tissue; CSA, cross-sectional area; CVD, cardiovascular disease; MT, muscle tissue; $\mathrm{HU}$, Hounsfield unit.

fat accumulation. The international scope of the study also supports the generalizability of the data. Body composition analysis was performed rigorously, according to standardized and recommended procedures. ${ }^{13}$ There are however potential limitations that need to be considered in interpreting the data. Despite the relatively large study sample and the 3-year follow-up, the number of deaths $(\mathrm{n}=50)$ was likely too small to secure the notion of a possible relationship between ectopic fat accumulation and survival in COPD. Because ECLIPSE CT scans were not performed purposely to determine body composition, the analysis had to be restricted to those participants with abdominal CT images, which would be valid for segmentation of AT and muscle area and densities. We do not believe, however, that these issues compromise the validity of our findings because baseline characteristics of patients involved in this substudy were similar to those of the entire ECLIPSE cohort. ${ }^{12}$

ECLIPSE did not mandate to match control subjects with patients with COPD. As such, important differences in age, sex distribution and smoking status were seen between the two groups. This issue was addressed by controlling for baseline differences for these variables in all analyses. In addition, physical activity levels were not assessed, making it difficult to comment on the potential impact of sedentary behaviors on the body composition differences observed in COPD in this cohort. Although ECLIPSE was a considerable undertaking, it remains that 3 years of follow-up was likely too short to highlight the clinical impact of body composition abnormalities, particularly in this cohort of patients with advanced COPD. Finally, some important well-established cardiovascular risk factors such as lipid and glycemic profile were not assessed. In this regard, it could be argued that these traditional risk factors could at least partly capture the elevated cardiovascular risk in COPD.

\section{Conclusion}

Our study adds to the existing knowledge by confirming the presence of increased ectopic fat accumulation in COPD. 
In this patient population, body composition abnormalities in the form of increased CSA of VAT and fat infiltration of intraabdominal muscle and reduced CSA of intra-abdominal muscle were associated with cardiovascular comorbidities, diabetes and gastroesophageal reflux as well as with relevant clinical outcomes such as walking capacity and $\mathrm{FEV}_{1}$ decline. As successfully accomplished in other patient populations, we submit that assessing the level of ectopic fat accumulation is relevant in COPD. In addition, we suggest that we should also consider the development of lifestyle modification programs focusing on both nutritional quality and physical activity/exercise with the objective of improving AT distribution/ectopic fat in patients with COPD.

\section{Acknowledgments}

The authors thank Véronic Tremblay for the body composition analysis platform of the Institut universitaire de cardiologie et de pneumologie de Québec for ensuring standardization of the CT images analyses and Gaétan Daigle for statistical assistance. They also thank all the subjects, investigators and study site staff who participated in ECLIPSE. This study was funded by GlaxoSmithKline (GSK). MM was a recipient of a research training award from the Fonds de la recherche Québec - Santé. FM holds a GSK/Canadian Institute of Health Research (CIHR) Research Chair on COPD at Université Laval. $\mathrm{J}-\mathrm{PD}$ is the scientific director of the International Chair on Cardiometabolic Risk, which is based at the Faculty of medicine, Université Laval. This work has been presented as an abstract at the 2015 meeting of the American Thoracic society.

\section{Disclosure}

MM, NA, IV, ER, MCW and JTM have no conflicts of interest to declare. JPD reports personal fees from Abbott Laboratories, AstraZeneca, GSK, Merck and Pfizer Canada Inc. and personal fees from Abbott Laboratories, Sanofi and Torrent Pharmaceuticals Ltd. outside the submitted work. EFMW reports personal fees from Nycomed, AstraZeneca, GSK and Novartis outside the submitted work. GRW reports other fees from GSK and other fees from Genentech outside the submitted work. HOC reports personal fees from GSK, grants from GSK during the conduct of the study, personal fees from Samsung and grants from Spiration Inc. outside the submitted work. WM reports personal fees and other fees from GSK, Pfizer, AstraZeneca and Boehringer Ingelheim, and grants from British Heart Foundation, Chief Scientist Office and Medical Research Council outside the submitted work. DDS reports personal fees from Amgen, grants and personal fees from AstraZeneca, personal fees from Boehringer Ingelheim and grants from Novartis outside the submitted work. FM reports grants and personal fees from Boehringer Ingelheim and GSK, grants from Nycomed and grants and personal fees from Novartis outside the submitted work. All fees are pooled with other revenues of the group of pulmonologists to which FM is a member and then shared among members of the group. The authors report no other conflicts of interest in this work.

\section{References}

1. Poulain M, Doucet M, Drapeau V, et al. Metabolic and inflammatory profile in obese patients with chronic obstructive pulmonary disease. Chron Respir Dis. 2008;5(1):35-41.

2. Sava F, Laviolette L, Bernard S, Breton M-J, Bourbeau J, Maltais F. The impact of obesity on walking and cycling performance and response to pulmonary rehabilitation in COPD. BMC Pulm Med. 2010;10(1):55.

3. Franssen FME, O’Donnell DE, Goossens GH, Blaak EE, Schols AMWJ. Obesity and the lung: 5. obesity and COPD. Thorax. 2008;63(12): 1110-1117.

4. van den Bemt L, van Wayenburg CAM, Smeele IJM, Schermer TRJ. Obesity in patients with COPD, an undervalued problem? Thorax. 2009;64(7):640; author reply 640-641.

5. Adams KF, Schatzkin A, Harris TB, et al. Overweight, obesity, and mortality in a large prospective cohort of persons 50 to 71 years old. N Engl J Med. 2006;355(8):763-778.

6. Sin DD, Man SF. Why are patients with chronic obstructive pulmonary disease at increased risk of cardiovascular diseases? The potential role of systemic inflammation in chronic obstructive pulmonary disease. Circulation. 2003;107(11):1514-1519.

7. van den Borst B, Gosker HR, Koster A, et al. The influence of abdominal visceral fat on inflammatory pathways and mortality risk in obstructive lung disease. Am J Clin Nutr. 2012;96(3):516-526.

8. Mathew MC. The effects of a smoking cessation intervention on 14.5-year mortality. Ann Intern Med. 2005;143(8):614-615. Author reply 615.

9. Calverley PMA, Anderson JA, Celli B, et al. Cardiovascular events in patients with COPD: TORCH Study results. Thorax. 2010;65(8): 719-725.

10. Després J-P, Lemieux I. Abdominal obesity and metabolic syndrome. Nature. 2006;444(7121):881-887.

11. Vestbo J, Anderson W, Coxson HO, et al. Evaluation of COPD longitudinally to identify predictive surrogate end-points (ECLIPSE). Eur Respir J. 2008;31(4):869-873.

12. Agustí A, Calverley PMA, Celli B, et al. Characterisation of COPD heterogeneity in the ECLIPSE cohort. Respir Res. 2010;11:122.

13. Després J-P, Ross R, Boka G, Alméras N, Lemieux I. ADAGIOLipids Investigators. Effect of rimonabant on the high-triglyceride/ low-HDL-cholesterol dyslipidemia, intraabdominal adiposity, and liver fat: the ADAGIO-Lipids trial. Arterioscler Thromb Vasc Biol. 2009;29(3):416-423.

14. Agustí A, Edwards LD, Rennard SI, et al. Persistent systemic inflammation is associated with poor clinical outcomes in COPD: a novel phenotype. PLoS One. 2012;7(5):e37483.

15. Coxson HO, Dirksen A, Edwards LD, et al. The presence and progression of emphysema in COPD as determined by CT scanning and biomarker expression: a prospective analysis from the ECLIPSE study. Lancet Respir Med. 2013;1(2):129-136.

16. Alluri K, Joshi PH, Henry TS, Blumenthal RS, Nasir K, Blaha MJ. Scoring of coronary artery calcium scans: history, assumptions, current limitations, and future directions. Atherosclerosis. 2015;239(1): $109-117$. 
17. Global Initiative for Chronic Obstructive Lung Disease [homepage on the Internet]. Global Strategy for the Diagnosis, Management and Prevention of COPD. 2015:1-117. Available from: www.goldcopd. org. Accessed April 2015.

18. Kramer MS, Feinstein AR. Clinical biostatistics. LIV. The biostatistics of concordance. Clin Pharmacol Ther. 1981;29(1):111-123.

19. Fleiss JL. Reliability of Measurement. Hoboken, NJ: John Wiley \& Sons, Inc; 1986:1-32.

20. Spruit MA, Polkey MI, Celli B, et al. Predicting outcomes from 6-minute walk distance in chronic obstructive pulmonary disease. J Am Med Dir Assoc. 2012;13(3):291-297.

21. Vestbo J, Edwards LD, Scanlon PD, et al. Changes in forced expiratory volume in 1 second over time in COPD. N Engl J Med. 2011;365(13): 1184-1192.

22. Furutate R, Ishii T, Wakabayashi R, et al. Excessive visceral fat accumulation in advanced chronic obstructive pulmonary disease. Int $J$ Chron Obstruct Pulmon Dis. 2011;6:423-430.

23. Maddocks M, Shrikrishna D, Vitoriano S, et al. Skeletal muscle adiposity is associated with physical activity, exercise capacity and fibre shift in COPD. Eur Respir J. 2014;44(5):1188-1198.
24. Robles PG, Sussman MS, Naraghi A, et al. Intramuscular fat infiltration contributes to impaired muscle function in COPD. Med Sci Sports Exerc. 2015;47(7):1334-1341.

25. Yoshida Y, Marcus RL, Lastayo PC. Intramuscular adipose tissue and central activation in older adults. Muscle Nerve. 2012;46(5):813-816.

26. Goodpaster BH, Thaete FL, Simoneau JA, Kelley DE. Subcutaneous abdominal fat and thigh muscle composition predict insulin sensitivity independently of visceral fat. Diabetes. 1997;46(10):1579-1585.

27. Dallman MF, la Fleur SE, Pecoraro NC, Gomez F, Houshyar H, Akana SF. Minireview: glucocorticoids - food intake, abdominal obesity, and wealthy nations in 2004. Endocrinology. 2004;145(6):2633-2638.

28. Vestbo J. Systemic inflammation and progression of COPD. Thorax. 2007;62(6):469-470.

29. Stockley RA. Progression of chronic obstructive pulmonary disease: impact of inflammation, comorbidities and therapeutic intervention. Curr Med Res Opin. 2009;25(5):1235-1245.

30. Diaz AA, Young TP, Kurugol S, et al. Abdominal visceral adipose tissue is associated with myocardial infarction in patients with COPD. Chronic Obstr Pulm Dis. 2015;2(1):8-16.

\section{Publish your work in this journal}

The International Journal of COPD is an international, peer-reviewed journal of therapeutics and pharmacology focusing on concise rapid reporting of clinical studies and reviews in COPD. Special focus is given to the pathophysiological processes underlying the disease, intervention programs, patient focused education, and self management protocols.

\section{Dovepress}

This journal is indexed on PubMed Central, MedLine and CAS. The manuscript management system is completely online and includes a very quick and fair peer-review system, which is all easy to use. Visit http://www.dovepress.com/testimonials.php to read real quotes from published authors. 